\title{
Interacting with Digital Media at Home via a Second Screen
}

\author{
Dr Emmanuel Tsekleves ${ }^{1}$, Dr Leon Cruickshank ${ }^{2}$, Dr Annette Hill ${ }^{3}$, Dr Koko Kondo ${ }^{3}, \mathrm{Mr}$ \\ Roger Whitham ${ }^{1}$ \\ ${ }^{1}$ Brunel University School of Engineering and Design, Uxbridge, UB8 3PH, UK \\ ${ }^{2}$ Lancaster University, Institute of Contemporary Arts, Lancaster, LA1 4YW, UK \\ ${ }^{3}$ Westminster University, School of Media, Arts and Design, Northwick Park, Harrow, HA1 3TP, UK \\ Contact: Emmanuel.Tsekleves@brunel.ac.uk
}

\begin{abstract}
Interacting with digital media is at the epicentre of every household. In recent years Interactive Television (iTV) has become a household technology on a global scale. However, iTV is still a new technology in the early stages of its evolution.

Our previous research looked at how everyday users of iTV feel about the interactive part of iTV. In a series of studies we investigated how people use iTV services; their likes, dislikes, preferences and opinions. We then developed a second screen-based prototype device in response to these findings and tested it with iTV users in their own homes.

This is a work in progress paper that outlines the work carried previously in the area of controlling interactive Television via a second screen. The positive user responses led us to extend the scope of our previous research to look into other related areas such as barriers to digital interactive media and personalisation of digital interactive media at home.
\end{abstract}

The paper is organised into three sections. The first section presents the main aims and objectives of our previous and current research work. The second section provides an outline of our previous research methodology, prototype systems and results. The third section provides the reader with information on the progress of our research endeavours upto-date and discusses some of the main findings of the first user study conducted thus far.

\section{Research Aims and Objectives}

The advent of interactive Television enables TV users to access new type of services from their TV sets but also brings into the forefront several issues and design challenges. These include technological issues with realising the ideas of the interface designer, to economic models to the often-unexamined reaction of users to interactive services.

Our research work can be seen as two individual projects.

The User-led Interactive Television (UITS) project aims at addressing several of the issues identified above by conducting a number of empirical studies and using these results within a knowledge transfer network bringing together specialists from industry, technological research, empirical user research and the users themselves. This project has been completed and several of its findings have been published in [1], [2].

Barriers to Interactive Television (BITe) is a two years project that builds on the success of the UITS project and is in collaboration with the University of Westminster.

BITe focuses on two key issues: barriers to digital interactive media and personalisation of digital interactive media. These apply to both creation of media and its use in the home. Personalisation includes personalising media products such as electronic programme guides or mobile phones, and creative production of photos and music for exchange, and websites. These issues pose considerable challenges for both technically and in terms of usability. 
In the BITe project, there is a total three of user studies planned involving 30 households in the Greater London area, with access to digital television and broadband, containing a balance of ages, gender, and socio-economic status. They aim to address digital media and everyday life, barriers to digital media, personalisation of digital media.

Based on a user-led methodology [2], [3] practical proposals addressing barriers to digital media, and personalisation of digital media, as collected from the above user studies will be developed will be tested and revised with the user studies' participants. This will take place during the fourth and last visit, which will form a usability testing of new products.

Some of the issues that will be addressed are:

- What are the barriers to using digital media, including digital television, PVRs, DVD recorders, internet, mobile phones, I-Pods, digital cameras?

- In what ways can people further engage and participate with digital interactive media?

- How do people personalise digital interactive media, and does this varies according to age and gender and socio-economic status?

\section{Previous Work}

This section presents the work that has been carried in the UITS project. This involved a similar to the BITe project approach and methodology, as described above.

More precisely, in winter of 2005 an initial study involving 20 households in the Greater London area was conducted. This included indepth interviews with household members about the information services participants interacted with. Participants were asked a wide range of questions about their use of interactive Television and were observed interacting with their televisions.

A total of 62 participants were interviewed in our initial study with ages ranging from 14 to over 50 and from a wide range of socioeconomic backgrounds. All households involved in our study subscribed to either Sky or Telewest digital services.

\subsection{Initial Results}

The key findings of our initial study can be outlined in the following:

- Interactivity is a dirty word.

Interactive features of iTV services are associated with commercialisation, hidden costs, security risks, technical problems, poor customer services and time wasting. While younger participants were less disposed to these connotations, interactive features were rarely viewed in an entirely positive light. The study also revealed that interactivity is only perceived as a positive medium when it is safe and secure. As Anne says: "interactivity can be a good thing, but only if it is safe", indicating risks to using new technology. This perception was also echoed by several other respondents too. For instance Richard states that "I don't think I'll use it for playing games or gambling", raising his concern about the security issues involved with the new technology.

- A focus on the familiar.

Study participants generally made limited use of the wide array of interactive features available. Instead they concentrated on the most familiar and essential functions. While younger household members often mastered many interactive features of their iTV services the older generation tended to be must less experimental. The Electronic Program Guide (the program schedule available on the TV itself) was a feature used by nearly all participants, while specialised interactive features unique to specific TV programs or channels were rarely used. The reminder function was also very popular amongst viewers as it was thought to be easy to use. According to Mark:

"I like that a lot, I can never be bothered with the guide... I just set the reminder, and then it comes on three minutes before the programme starts"

- Hidden costs.

Participants frequently expressed concerns about hidden costs and security risks encountered when using services available through iTV. Adult participants were especially worried about their children entering competitions through iTV services without being aware of the costs involved. Many families involved in our study could recount occasions when they had been received large bills without having been aware of the costs they had incurred. Marry, a housewife decided not to use the interactive facilities of the digital 
TV box, after her family was overcharged for consuming interactive services.

"We don't use the interactive so much. We've got troubles on the phone line. Coz we've got the phone init, we've got also internet init'.

- $\quad$ Speed and simplicity.

A recurring theme in our study's results is the desire for rapid and direct access to content. The interactive functions participants most favoured were those which facilitated rapid access to content with a minimum of fuss. Most participants liked the 'now and next' program listing which appears at the bottom of the screen because it is fast to use and easy to dismiss. In comparison the $\mathrm{A}$ to $\mathrm{Z}$ channel listing was disliked because it takes up all the screen and is often slow to navigate.

\subsection{Prototypes}

The initial study provided a plethora of data to inform the development of prototypes that were evaluated in a second study. In particular we wished to investigate the possibility of employing a second screen to mediate interaction with iTV services. To investigate this we designed a touch-screen remote control device capable of supporting all of the interactive functions currently available only on the TV itself. There have been very few projects and research work that focuses on the control and interaction of the iTV via a PDA with [4], [5] and [6] being the most prominent and complimentary to our research work. In addition to this there have been projects associated with the use of touch pads (but not touch sensitive display screens) as part of remote controls [7] and in using PDAs to control household electronics using both stylus and speech input [8]. There have also been projects that control TV in a multi-user context with mobile phones [9].

Our objectives were to create a prototype that simulated the iTV experience as closely as possible within a study participant's home. This included the integration of live Freeview TV (a free to air terrestrial digital television service in the UK) [10] in the system. As we were developing a new User Interface (UI) approach to the EPG and services we decided to create a parallel service, mirroring the UI design used in the PDA but optimised for use with a conventional iTV infrared remote. This we call the 'onscreen' prototype as the dynamic portion of the UI is represent on the TV screen, just like existing commercial iTV systems.

\subsubsection{The On-screen Prototype}

Taking existing commercial systems as a basis for the interaction model, the on-screen prototype was design to be analogous to the systems used by our study participants. To make the on-screen prototype as usable as possible we also aimed to solve a number of key usability problems with onscreen iTV UI identified in our first study.

The on-screen prototype employed almost the same hardware as the PDA prototype. To this we added a USB infrared receiver and a generic iTV remote to allow our prototype laptop to function just like a set top box.

\subsubsection{The PDA Prototype}

The objectives of the PDA prototype design were to ensure the simplicity and immediacy of the common actions of iTV operation including, volume adjustment, channel switching and EPG access. The PDA needed to match the strengths of existing remote controls wherever possible. Additionally we wished to introduce new features suggested to be desirable by our initial study. These included setting reminders, setting recordings, channel history, customising the position of channels in the EPG, and pay-as-you-go billing. While most of these features are available in existing commercial systems such features often require complex interactions for use, something we wanted to avoid in our prototype.

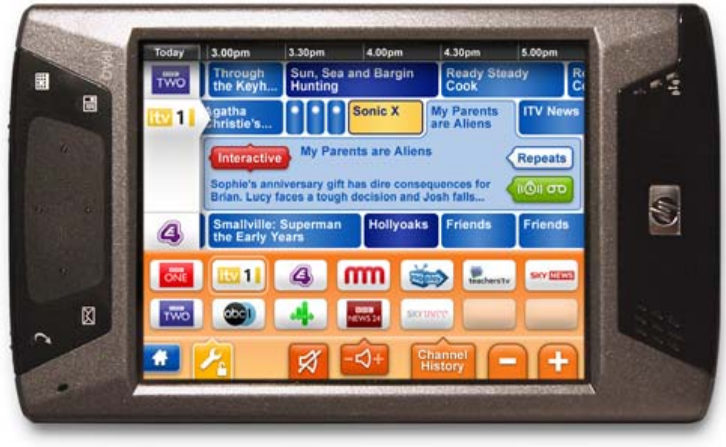

Figure 1: The PDA Prototype displaying the EPG

We also decided to support secondary PDA devices in our study. This would offer an EPG and access to iTV services, but without control over channel switching or audio. Our secondary unit would allow simultaneous consumption of iTV services from independent PDA terminals. 


\subsubsection{Hardware}

We used two HP iPAQ hx4700 Pocket PCs for the test. These have a 4-inch backlit screen with a resolution of $640 \times 480$ pixels and 64 thousand colours. The device was selected for the high quality of its screen, with a physical pixel resolution of 200ppi (pixels per inch), compared to typically less than 100ppi for a computer monitor and less than 50ppi for a standard definition television. This provides an excellent platform for visualisation of the UI, allowing for crisp, legible text at physical sizes that would not be possible on television screens.

The hx4700 has integrated $\mathrm{WiFi}$ and Bluetooth, this allowed the PDA to communicate with a laptop taking the place of a set-top box with live multi-channel Digital TV Freeview broadcast in real time. The communication between the PDAs and the laptop is supported by two Bluetooth devices on the hardware level and by a Java socket server application on the software level. Both live TV and the service simulation are outputted from the laptop to the participant's own television set increasing the sense of realism.

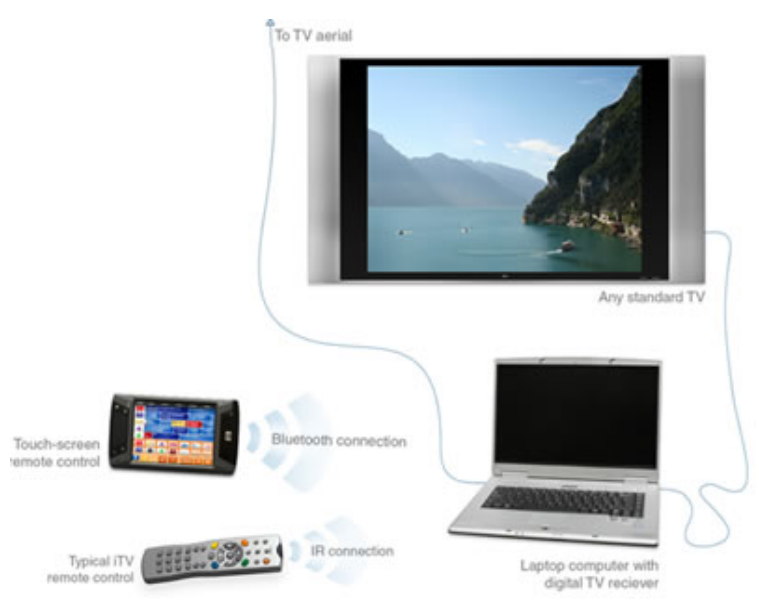

Figure 2: The On-screen and PDA Prototypes

The EPG portion of the prototype updates automatically using standard XML TV metadata [11] available from the Bleb.org website [12].

\subsection{Prototype Evaluation}

In the spring of 2006 we returned to the 20 households involved in our initial study to test the prototypes we had developed. Each visit lasted 1-2 hours and involved participants trying out the two prototypes on their own TVs and talking about their feelings towards them.

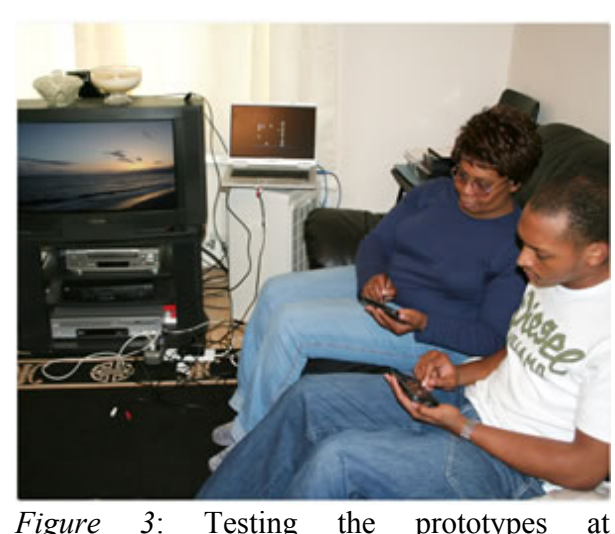
participants' homes

Common themes from our evaluation can be outlined in the following:

- $\quad$ Ease of use.

Almost all participants responded very positively to the touch-screen remote. Women and teenagers of both sexes were especially enthusiastic about the simplicity of the device and the ease with which it could be used: "I think it makes more clear what is going on...I think it's quite easy to use" Anne

"I think it's just really straightforward" Linda "I prefer this to the one on the screen, I'd prefer to have this" Marry

\section{- $\quad$ Multiple users.}

Participants were positive about the unobtrusive access the touch-screen remote allowed to program listings and other services: individuals could see what was on TV and set reminders without needing to intrude on the main TV picture.

\section{- $\quad$ Speed and efficiency.}

Study participants appreciated the speed of both the touch-screen and the conventional prototypes.

- Concerns with cost.

Most study participants were weary of the cost a touch-screen device such as our prototype might have. Parents were particularly worried about such a device getting damaged in the hands of young children: "I think it's expensive for people to buy that", Peter

"If you loose the remote control [it would cost you $£ 10]$, if you loose this it'll cost you a lot" Mark 


\section{- Parental control}

The possibility of having multiple touch-screen remote controls, each customised for the need of an individual of group, appealed to parents wishing to control what their children could watch. The portability of the touch-screen remote would allow parents to keep an eye on what their children were watching from another room in the house.

One should also mention that there was doubtless a novelty factor at play when participants evaluated the PDA against the more traditional on-screen UI. This could be minimized by leaving the prototypes within the household for a week, to allow users to interact more. For this reason in the BITe project we aim to incorporate this in our usability testing.

\section{Current Work}

At present we are close to the end of the first year of the BITe project. We have conducted the first of the three scheduled user studies and are currently conducting the second one. These will enable us to gather useful data before developing a new prototype that will be tested in the fourth and last visit at the participants' homes.

The first user study involves 30 households in the Greater London area. This is an exploratory study and employs interviews and open-questionnaires, aiming at investigating participants' digital media experiences. A preliminary analysis of the data collected thus far has revealed some interesting results and are summarised below:

\section{- $\quad$ Media Use and Experiences}

The purpose and use of the Internet varies according to age. Participants who use Personal Computers (PC) at work do not regard the Internet (or use the computer) as a relaxing experience, whereas children regard it as 'entertainment' since they cannot use the computers at school to consume media. All media experiences for children become 'purely entertainment' (except when using it for homework). Parents on the other hand regard it more as a practical tool in their life for shopping research, job seeking, work related activities, banking, emailing friends and family abroad. For most adults and parents television is seen as the principle entertainment medium.

- Multi-platform Media

Most of the participants expressed a positive view on multi-platform media. They think having different media sources is very good, including old media such as magazines and radio especially for news and information gathering, in case of the adults. Teenagers and children tend to use these mostly for playing games from related websites- such as the Cartoon network or Disney channel, where they can relate with the characters in the games.

Despite the positive view on multi-platform media, users do not access the Internet from their mobile phone, and portable media devices. This is due to users' uncertainty of the actual costs and due to the limited number of websites that are being specifically designed to run on a mobile environment (scaling, design and performance). For teenagers and especially female ones, having a new telephone seems to be the most desirable act (more than owning iPod or a game console).

\section{- Creative Use of Media}

Although most of the households own video camera and digital camera, they just have raw material without editing or developing photos due to lack of skills, time, and memory in their PC.

Most of the users want to be able to make their own websites where they can upload photos, however, they do not know how to do so. Teenagers admitted having tried to create their own websites. However some of them had negative experiences by uploading their own websites due to negative and often 'nasty' comments from their peers. Others think that it is a boring process to make websites, even though their friends often do create their own websites. People including children want to upload their video clips and photos over YouTube and other web sites, but do not know how to achieve that. They often lack the knowledge as to which website they should use, or how they should upload their photos on the web.

We want to further test our assertion that using a PDA for control can address several of the issues raised by our test subjects. A PDA offers the opportunity to remove the need to show UI elements on the main television screen. This avoids many of the problems related to UI latency/lack of reaction speed in iTV systems as viewers are not going to miss broadcast information because an interactive service is not responding rapidly enough (e.g. missing a goal in a football match). 
A PDA approach also offers the opportunity to extend the model of iTV operation by offering a far richer interaction mechanism than conventional remote controls. More than one PDA can access iTV services at the same time such that a number of users can be voting, browsing the EPG and setting programmes to be recorded at the same time. Personalisation either locally on each terminal (each user has his/her own PDA with customised UI and/or hardware features) or virtually (users log-on to their own customized setting stored on the same PDA) is possible. This also allows for the dynamic reconfiguration of the UI to reflect the users needs and desires.

This is not to say that PDA usage is without UI drawbacks, particularly with respect to the small screen, the lack of a full-sized keyboard and the lack of tactile feedback from the screen which practically negates the possibility of operation without needing to look at the device for feedback.

\section{Conclusion and Future Work}

We have presented a work in progress paper that has outlined the main results of the deployment and use of a second screen approach to help users to control and consume iTV media in a home environment.

The second screen prototype system we have developed and tested offers significant advantages over conventional remote controls when consuming iTV services. Users show a strong preference for this system over conventional remotes, primarily due to the mobility of the PDA and its superior visualisation of service information. By using the PDA for TV control and as a terminal for iTV service consumption we provide an uninterrupted broadcast experience on the TV itself, addressing an important concern highlighted in our first study, while providing superior interaction with iTV services for PDA users. This also allows multiple individuals to consume interactive content simultaneously.

Reservations about the PDA system were recorded, linked both to the perceived cost of the units and the difficulty of finger-based interaction with the touch screen. Many of these problems appear to lie in the UI design and their resolution is a goal of future work.

Based on the positive responses we have continued research in a new project that seeks to expand this paradigm beyond iTV to other interactive digital media at home. This project will focus on the key issues of barriers to digital interactive media and personalisation of digital interactive media. Lastly, several results of the preliminary analysis of the first user study of our current research work have been also presented here.

\section{References}

[1] Cruickshank L.,. Tsekleves E, Hill A., Kondo K., Whitham R. (2007) A User Led Examination of the Barriers to Entry for Interactive TV User Experience Magazine, Volume 6, Issue 3, Usability Professionals' Association

[2] Cruickshank L., et al. (2006) User Led Design and Production of Interactive TV Services. Wonderground: 2006 Design Research Society International Conference. Lisbon: Portugal. November 2006

[3] Green, et al. 2004. Capturing use requirements for an integrated home environment. In ACM Proceedings of the third Nordic conference on Human-computer interaction. Tampere, Finland 2004.

[4] Robertson, S., et al (1996) Dual device user interface design: PDAs and interactive television, Proc. CHI '96, pp79-86.

[5] Cesar P., et al (2007) An Architecture for Nonintrusive User Interfaces for Interactive Digital Television. Lecture Notes in Computer Science: Interactive TV: a Shared Experience. Volume 4471. Heidelberg: Springer-Verlag Berlin, pp 11-20

[6] Ma, M., et al (2004) IDTV broadcast applications for a handheld device. Proceedings of IEEE International Conference on Communications. IEEE, June 2004. pp 8589

[7] De Vet, J., Buil, V. (1999) A personal digital assistant as an advanced remote control for audio/video equipment. Proc. of the $2^{\text {nd }}$ Workshop on HCI with Mobile Devices.

[8] Nichols, J., et al (2002) Generating remote control interfaces for complex appliances. Proc. UIST' 02 , pp 161-170.

[9] Park, J., et al (2006) Sharable digital TV: relating ethnography to design through un-useless product suggestions. Proc. CHI '06, pp 11991204.

[10] Freeview 2007. Retrieved July, 2007, from http://www.freeview.co.uk

[11] XML TV 2007. Retrieved July, 2007, from http://xmltv.org/wiki/

[12] Bleb.org 2007. Retrieved July, 2007, from http://www.bleb.org/tv/ 